\title{
Mateja PETROVČIČ
}

\section{Introduction}

This book was published in the Linguistics Series of European University Studies, and is written in German. As the book's title suggests, this monograph is primarily a comprehensive analysis of reflexivity in spoken Standard Chinese in the framework of Integrational Linguistics. ${ }^{1}$ The author demonstrates that Chinese marks reflexivity only phonologically, with the use of reflexive pronoun(s), and argues that ziji (自己) is the only reflexive pronoun in Standard Chinese.

Different languages distinguish between referential and non-referential reflexive pronouns, and the author briefly demonstrates this with German sich. Referential uses denote semantic reflexivity (inhaltliche Reflexivität), whereas non-referential uses represent formal reflexivity (formale Reflexivität). Su asserts that the Chinese reflexive pronoun ziji is always referential and that there is no formal reflexivity in Chinese.

Since the research mainly focuses on the word ziji, not only in its reflexive usage but also in relation to intensifying and contrastive meanings and effects, this monograph could also be considered as a comprehensive research on ziji in Standard Chinese.

\section{Summary}

The volume is organized into five sections (A-E) and numbered chapters (0-16), followed by two appendixes written by Hans-Heinrich Lieb, the founder of the Integrational Linguistics.

The book opens with an introductory chapter, outlining the subject and scope of research, explaining the reason for the author's specific selection of examples, and sketching the structure of the book.

\footnotetext{
${ }^{1}$ The term "Integrational Linguistics" refers to the theory developed by Hans-Heinrich Lieb and should not be confused with the approach proposed by Roy Harris. (Sackmann, 2000, p. 472)
} 
Chapter 1 presents the issue of reflexivity in Chinese, from the distributional properties of ziji to its intensifying and contrastive effect. The author also raises the question of how many reflexive pronouns there are in Chinese. Two concepts relevant to further discussion are introduced at this stage, i.e. long-distance binding and blocking effect. This is linked to the problem of identifying the antecedent of the reflexive pronoun ziji, especially in situations when more possible antecedents appear in a sentence.

Chapter 2 gives a brief overview of the relevant research history; Chapter 3 focuses on the antecedent identification in the scope of generative linguistics; Chapter 4 finally closes the Section A with the findings in more semantic, pragmatic or functional oriented theoretical frameworks.

Sections B to E focus on Integrational Linguistics. Chapters 5 to 7 first introduce the reader to this theoretical framework in general and point out some properties of Chinese idiolect systems, which the author considers relevant for the following discussion.

Section $\mathrm{C}$ then starts with the actual investigation of reflexivity in Chinese. Chapter 8 deals with the concepts of reflexivity and intensification related to ziji. Author argues that reflexivity in Chinese should not be understood on the word-level of the reflexive pronoun ziji, but on the sentence-level. Su also stresses that the reflexive pronoun ziji should be clearly distinguished from its homophone, the intensifier ziji with completely different properties.

Because sentence-level is relevant for the antecedent selection, Chapter 9 first provides some assumptions and definitions of predicate, subject and object in Chinese. The author further stresses the difference between ellipsis (Ellipse), empty complement (leeres Komplement) and complement suppression (Komplementunterdrückung). Related to the above questions are also copula verbs, relational verbs and pivotal constructions in Chinese, which are of considerable importance for further discussion and described in the last parts of Chapter 9.

Chapter 10 briefly analyses sentences with the particles $b a$ (把), bei (被) and the three $d e$ 's (的, 得 and 地). A more detailed investigation of Chinese ba/bei sentences in the scope of integrational linguistics is carried out in the appendix B at the end of the book.

Section D investigates highly controversial problem in Chinese, i.e. how an antecedent of the reflexive pronoun ziji is selected when several potential antecedents appear in the sentence. This is a difficult task in a language like Chinese with no pronoun-antecedent agreement. The author limits her discussion to the carefully selected examples with just one reflexive ziji and maximal three potential antecedents. Chapter 11 focuses on antecedent identification for subjects, Chapter 12 links to antecedent identification for objects, while Chapter 13 takes under consideration some restrictions for antecedent selection. Chapter 14 further discusses sentences with blocking effect mentioned already in Chapter 1. 
Section E (Chapters 15 and 16) is the concluding part of Su's research. Chapter 15 summarizes all significant findings and conclusions, whereas Chapter 16 presents her hypotheses about reflexivity in every idiolect system.

The book is written for general linguists, preferably interested in integrational linguistics, experts in Standard Chinese, researchers of other languages who are working in the area of reflexivity, and is also a valuable piece of work for contrastive studies comparing closely related features such as reflexivity, intensification and contrast in a language.

\section{Evaluation}

This monograph represents an exceedingly detailed description of reflexivity in Chinese and deserves a readership far beyond the German academic community. The author very systematically introduces the reader to the theoretical framework and some basics of Chinese language, so readers with not much knowledge of Standard Chinese can also understand and benefit from this book.

Reflexivity in language is a complex and challenging issue, which is difficult to present in a simple linear way. However, the author skillfully interweaves various theoretical concepts and actual usages of the reflexive pronoun ziji, starting with very simple sentences and gradually proceeding towards constructions that are more complex and even ambiguous.

To discuss a selected topic in a scope of a chosen theoretical framework is already an immense project. Su, however, was faced with the additional task of providing explanation for some other phenomena in Chinese, which are closely related to the issue of reflexivity, but have not been discussed in Integrational Linguistics. It might be observed from the References in this monograph and the homepage of Integrational Linguistics that only a few studies about Chinese language have been done so far. In order to provide a comprehensive explanation of ziji on the sentence-level, the author had to first define several crucial concepts such as predicate, subject, direct and indirect object (or in Su's terms "first" and "second object") in Chinese. Since there are already several definitions and interpretations of the concerned concepts in the existing literature, the presented explanation might receive critical feedback.

In the subchapter of personal and reflexive pronouns, the author provides a persuasive argumentation, why benren (本人), benshen (本身) and zishen (自身) should not be treated as reflexive pronouns, as was sometimes claimed in the previous literature. However, speaking of personal pronouns, the author does not even mention zanmen (咱们), although it could be easily defined in the same way as the other personal pronouns. It is unclear whether the author considers zanmen as an expression 
which does not belong to Standard Chinese, or is the omittance of this personal pronoun just a slip.

Throughout the entire book, the author provides numerous examples in Chinese. Moreover, from the Section $\mathrm{C}$ onward, most examples are also equipped with very illustrative structural diagrams. Their designing and formatting must have been a timeconsuming job.

A potential limitation of the volume is that almost all examples are intentionally very similar and rely on the author's language sense as a native speaker of Chinese. When striving for a detailed theoretical explanation, the author seems to overlook ambiguous meanings of some examples or applies slightly unconvincing explanations (e.g. 3.6/12.5). Although the development of author's hypotheses and related argumentations seem to be very plausible, it is desired that the findings are confirmed in more diverse contextual environments, for not only reflexive usage of ziji, but also for intensifying meaning and its contrastive effects.

On the whole, the monograph offers numerous contributions concerning the issue of reflexivity in Standard Chinese. From the perspective of Integrative Linguistics, the author develops a detailed new analysis of the Chinese ziji, that will undoubtedly afford linguists in this area insightful knowledge evoking future research.

\section{References}

Sackman, R. (Last modified: December 11, 2004). The Homepage of Integrational Linguistics. Retrieved August 10, 2011, from http://userpage.fu-berlin.de/sackmann/+en/main-en.html. Sackmann, R. (2000). Numeratives in Mandarin Chinese. In P. M. Vogel \& B. Comrie (Eds.), Approaches to the typology of word classes (pp. 421-478). Berlin: Walter de Gruyter. 